disease activity and organ damage were associated with impaired HRQoL in all aspects, while Asian patients reported better PCS scores (and $\beta=0.29 ; P=0.007$ ) and FACIT-Fatigue scores $(\beta=0.33 ; P=0.002)$.

Conclusion: BMI above normal was highly associated with HRQoL impairment, especially in physical aspects. Further survey to examine causality is warranted to support structured weight control strategies as an intervention towards a more favourable HRQoL.

Disclosure of Interests: None declared

DOI: 10.1136/annrheumdis-2020-eular.4016

\section{FRI0169 ANTINUCLEAR ANTIBODY SEROCONVERSION DURING FOLLOW-UP IN PATIENTS WITH SYSTEMIC LUPUS ERYTHEMATOSUS}

R. Gualtierotti ${ }^{1}$, G. Frontini ${ }^{2}$, F. Pregnolato ${ }^{3}$, P. Messa $^{2}$, G. Moroni ${ }^{2}$, P. L. Meroni ${ }^{3} .{ }^{1}$ University of Milan, Department of Medical Biotechnology and Translational Medicine, Milan, Italy; ${ }^{2}$ Fondazione IRCCS Ca' Granda, Ospedale Maggiore Policlinico, Nephrology Unit, Milan, Italy; ${ }^{3}$ IRCCS Istituto Auxologico Italiano, Immunorheumatology Research Laboratory, Cusano Milanino, Milan, Italy

Background: Systemic lupus erythematosus (SLE) is a chronic autoimmune disease characterized by the presence of autoantibodies and a variable spectrum of clinical manifestations and disease severity. The 2019 criteria for SLE classification by the American College of Rheumatology and European League against Rheumatism define ANA positivity by immunofluorescence or by an equivalent solid-phase assay as the entry criterion (1). However, the prevalence of ANA positivity and the reliability of solid-phase assays in SLE are still a matter of controversy (2). Furthermore, the significance of ANA negativisation during follow-up is uncertain (3).

Objectives: Our aim was to retrospectively analyse data on the frequency of ANA seroconversion during the follow-up in a cohort of SLE patients with renal involvement.

Methods: Adult patients independent of age at SLE onset with a follow-up duration of at least 36 months starting from January 2009 (for standardization of ANA measurement) and with at least one ANA measurement per year were included in this retrospective longitudinal study. Data on demographic, clinical and laboratory characteristics of the study population are reported in table 1. ANA have been measured with Hep2 cell immunofluorescence assay.

Table 1. Demographic, clinical and laboratory baseline characteristics of the 121 patients suffering from systemic lupus erythematosus (SLE).

\begin{tabular}{lc} 
Demographics & \\
\hline Gender, \%F $(n)$ & $93(112)$ \\
Age in years, mean $\pm S D$ & $41.6 \pm 12.6$ \\
Clinical features & \\
Age at SLE onset in years, mean $\pm S D$ & $28.0 \pm 11.9$ \\
SLE duration in years, mean $\pm S D$ & $13.8 \pm 9.5$ \\
SLEDAl, median (min-max) & $4(0-27)$ \\
Laboratory profile & \\
Serum creatinine mg/dL, median (min-max) & $0.8(0.4-2)$ \\
24h urine protein g/24h, median (min-max) & $0.5(0-13.8)$ \\
ANA, \%pos ( $n$ ) & $93(112)$ \\
Anti-ENA, \%pos (n) & $49(59)$ \\
Anti-dsDNA, \%pos $(n)$ & $43(51)$ \\
\hline
\end{tabular}

Results: A total of 121 SLE subjects with renal involvement were enrolled. Mean follow-up \pm standard deviation (SD) was $8 \pm 2$ years. Ten subjects $(8.3 \%)$ with positive ANA at the beginning resulted ANA negative at the end of the follow-up. These subjects had different initial ANA titres: 1:1280 $(n=1)$, $1: 640(n=2), 1: 320(n=2), 1: 160(n=3)$ and $1: 80(n=2) ; 48$ subjects $(39.7 \%)$ showed a decrease in ANA titre. Of the 9 patients (7.4\%) that were negative at the beginning of follow-up, 6 remained negative, whereas 3 showed ANA positivity at the end of the follow-up with ANA titres 1:160 $(n=2)$ and 1:320 $(n=1)$. No differences between subjects with and without ANA titre variations in terms of age $(p=0.551)$, disease duration $(p=0.786)$, SLEDAI at the beginning $(p=0.453)$ and at the end of follow-up $(p=0.169)$ were observed. ANA negativisation and titre variations at the end of follow-up did not correlate with any of the treatments taken during follow-up, including a history of cyclophosphamide $(p=0.788)$

Conclusion: In our cohort of patients with SLE and renal involvement, $10 \%$ of patients experienced negativisation and around $40 \%$ of patients showed a decrease in ANA titre during follow-up, independent of disease characteristics and previous treatment. Further studies are warranted to clarify the underlying mechanisms and clinical significance of ANA seroconversion and titre variation in SLE patients. However, based on our results, ANA positivity seems to be a relatively stable parameter further supporting its use as an entry classification criterion for SLE.

\section{References:}

[1] Aringer $\mathrm{M}$ et al. Arthritis Rheumatol. 2019; 71(9):1400-1412.

[2] Pisetsky DS et al. Autoimmun Rev. 2019; 18(12):102400.

[3] Frodlund $\mathrm{M}$ et al. Clin Exp Immunol. 2019. Epub ahead of print.

Disclosure of Interests: None declared

DOI: 10.1136/annrheumdis-2020-eular.2685

\section{FRI0170 THERAPEUTIC TARGETS AND QUALITY INDICATORS IN SYSTEMIC LUPUS ERYTHEMATOSUS (SLE), DEFINED ACCORDING TO THE 2019 UPDATE OF THE EULAR RECOMMENDATIONS: DATA FROM THE "ATTIKON" LUPUS COHORT}

K. Havatza ${ }^{1}$, K. Togia ${ }^{1}$, S. Flouda ${ }^{1}$, A. Pieta ${ }^{1}$, O. Gioti ${ }^{1}$, D. Nikolopoulos ${ }^{1}$, N. Kapsala ${ }^{1}$, A. Ntourou ${ }^{1}$, P. Rapsomaniki ${ }^{1}$, T. Gerogianni ${ }^{1}$, D. Tseronis ${ }^{1}$, M. Aggelakos ${ }^{1}$, T. Karageorgas ${ }^{1}$, P. Katsimpri ${ }^{1}$, G. Bertsias ${ }^{2}$, K. Thomas ${ }^{1}$ D. Boumpas', A. Fanouriakis'. "'Attikon" University Hospital of Athens, Rheumatology and Clinical Immunology, Athens, Greece; ${ }^{2}$ University Hospital of Heraklion, Rheumatology and Clinical Immunology, Athens, Greece

Background: Targets of therapy and quality of care are receiving increased attention in the management of SLE, as outlined in the 2019 update of the EULAR recommendations for SLE treatment.

Objectives: To assess compliance with quality indicators and attainment of treatment targets, according to recent EULAR recommendations, in the SLE cohort of "Attikon" Rheumatology Unit.

Methods: 100 consecutive SLE patients followed for at least one year were. A 30 item Quality Indicator Set (QIS) was developed, according to the 2019 EULAR recommendations for SLE, to include laboratory tests for diagnosis and monitoring, evaluation of disease activity and damage using validated indices, use of patient-reported outcomes, counselling for women's health and reproduction issues, attainment of targets of therapy [remission or low disease activity state (LLDAS) with low-dose glucocorticoids (GC, $\leq 7.5 \mathrm{mg} /$ day prednizone) and hydroxychloroquine ( $\mathrm{HCQ}$ dose $\leq 5 \mathrm{mg} / \mathrm{kg} /$ day)], prevention of disease flares and prevention and management of co-morbidities. Chart review and patient interview was performed to assess the degree of compliance with each item of the QIS and achievement of treatment targets.

Results: Disease activity was monitored by means of validated indices in 31\% and antiphospholipid antibody testing during the first 6 months from diagnosis was performed in $58.8 \%$ of patients. Sustained remission (defined as remission of a sustained period of 12 months) or LLDAS was achieved by only $3 \%$ and $22 \%$ respectively; in contrast, other targets of therapy, such as $\leq 1$ minor flares during last year, were achieved by $85 \%$ ( $43 \%$ had complete absence of flares), with $90.2 \%$ of patients receiving low-dose GC and $81.8 \%$ corrected HCQ dose. Fertility and pregnancy counselling were offered in $40 \%$ (12/30 eligible women) and $63.3 \%(19 / 30)$ of patients, respectively, while $65.4 \%$ had a Pap Test and only 3 of 32 eligible patients had received the HPV vaccine. Annual lipid status was assessed in $43 \%$ and counselling for smoking cessation in $44.6 \%$. Flu vaccination was performed in $77 \%$, while pneumococcal (including both of the pneumococcal vaccines) and herpes-zoster vaccination, were given in $32.7 \%$ and $2 \%$ (1/44 eligible patients) respectively.

Conclusion: Our real-life data suggest low vaccination rates (excluding flu) and suboptimal management of cardiovascular risk factors in lupus patients. While the majority of patients received the suggested doses of GC and HCQ, only one quarter of patients achieved remission or LLDAS. There is an unmet need for new therapies in SLE to improve therapy targets.

\section{References:}

[1] Arora S, Sequeira W, Yazdany J, Jolly M, "Does Systemic Lupus Erythematosus Care Provided in a Lupus Clinic Result in Higher Quality of Care Than That Provided in a General Rheumatology Clinic?", Arthritis Care Res. 2018 Dec;70(12):1771-1777. doi: 10.1002/acr.23569. Epub 2018 Nov 10.

Disclosure of Interests: KATERINA HAVATZA: None declared, KONSTANTINA TOGIA: None declared, Sofia Flouda: None declared, Antigoni Pieta: None declared, Ourania Gioti: None declared, Dionysis Nikolopoulos: None declared, Noemin Kapsala: None declared, Aliki Ntourou: None declared, Panagiota Rapsomaniki: None declared, Thaleia Gerogianni: None declared, Dimitrios Tseronis: None declared, Michail Aggelakos: None declared, Theofanis Karageorgas: None declared, PELAGIA KATSIMPRI: None declared, George Bertsias Grant/research support from: GSK, Consultant of: Novartis, Konstantinos Thomas: None declared, DIMITRIOS BOUMPAS Grant/research support from: Unrestricted grant support from various 
pharmaceutical companies, Antonis Fanouriakis Paid instructor for: Paid instructor for Enorasis, Amgen, Speakers bureau: Paid speaker for Roche, Genesis Pharma, Mylan

DOI: 10.1136/annrheumdis-2020-eular.6255

\section{FRI0171 THE CHANGES OF IMMUNE FUNCTION AND CLINICAL INDEXES WITH SYSTEMIC LUPUS ERYTHEMATOSUS AFTER IMMUNOREGULATORY COMBINATION THERAPIES}

X. Liu' ${ }^{1}$, X. Liü ${ }^{2}, \mathrm{H} . \mathrm{Hou}^{3}, \mathrm{X} . \mathrm{LI}^{1}$ on behalf of Li Xiaofeng Team. ${ }^{1}$ The Second Hospital of Shanxi Medical University, Taiyuan, China; ${ }^{2}$ Taiyuan University of Technology, Taiyuan, China; ${ }^{3}$ Inner Mongolia Normal University, Huhehaote, China

Background: Recent studies have reported that some drugs such as low-dose interleukin-2, rapamycin, metformin, retinoic acid and coenzyme Q10 could promote the proliferation and functional recovery of regulatory T cells (Treg) in patients with autoimmune diseases. However, the effects on the balance of Treg cells and pro-inflammatory lymphocytes and long-term efficacy have rarely been reported.

Objectives: To evaluate the changes of peripheral lymphocyte subsets, conventional drugs and remission rate in patients with systemic lupus erythematosus (SLE) after immunomodulatory combination therapies.

Methods: A total of 189 patients with SLE from the Second Affiliated Hospital of Shanxi Medical University from January 2016 to October 2019 were enrolled, who were divided into well-controlled group and untargeted control group taking a full consideration of the patient's symptoms, signs and related laboratory findings. We measured the absolute counts of $\mathrm{B}, \mathrm{NK}, \mathrm{CD} 8+\mathrm{T}$ and helper T 1 (Th1), helper T 2 (Th2), helper T 17 (Th17) and Treg cells in peripheral blood of patients before immunomodulatory combination therapies and during the 3 months and 6 months of follow-up and 190 sex- and age- matched control individuals using flow cytometry. Moreover, the ratios of various cells to Treg cells were calculated. Results: Compared with healthy controls, Treg cells in SLE patients were significantly lower before the treatment with immunomodulator, while the ratios of various pro-inflammatory lymphocytes to Treg cells (such as Th2/ Treg, Th17/Treg, CD8+T/Treg, etc.) were higher. After 3 months and 6 months with immunomodulatory therapy, the absolute number of Treg cells in peripheral blood of SLE patients increased obviously reaching to normal level. Accordingly, the ratios of various pro-inflammatory lymphocytes to Treg cells recovered. At the same time, the dose of glucocorticoid and disease-modifying antirheumatic drugs (DMARDs) decreased distinctly. Additionally, the well-controlled group was able to maintain a high remission rate, and the untargeted control group could achieve a higher response rate after immunomodulatory treatment.

Conclusion: The imbalance between pro-inflammatory lymphocytes and Treg cells caused by the significant decrease of Treg cells may be the main cause of SLE. And immunomodulatory combination therapies we came up with may reverse the imbalance of proinflammatory lymphocytes and Treg cells, which is an potential and effective treatment for SLE.

References:

[1] Noack M, Miossec P. Th17 and regulatory T cell balance in autoimmune and inflammatory disease[J]. Autoimmun Rev, 2014, 13(6): 668-677.

[2] Yu A, Snowhite I, Vendrame F, et al. Selective IL-2 responsiveness of regulatory $T$ cells through multiple intrinsic mechanisms supports the use of low-dose IL-2 therapy in type 1 diabetes. Diabetes. 2015;64: 2172-2183.

[3] Schuiveling M, Vazirpanah N, Radstake TRDJ, Zimmermann M, Broen JCA. Metformin, A New Era for an Old Drug in the Treatment of Immune Mediated Disease?[J]. Curr Drug Targets, 2017;18:1-15.

Table1. The changes of remission rate in the no-remission group during follow-up.

\begin{tabular}{lllll}
\hline Follow-up period & Total patients & Remission & No-remission & Remission rate(\%) \\
\hline Baseline & 92 & 0 & 92 & 0 \\
3 Months & 72 & 33 & 39 & $45.8^{\mathrm{a}}$ \\
6 Months & 74 & 42 & 32 & $56.8^{\mathrm{a}}$ \\
\hline
\end{tabular}

a: Compared with baseline; b: Compared with 3 months.

Acknowledgments: We would like to express our sincere gratitude to all our coworkers and collaborators, Jing Luo, Xiangcong Zhao, Chen Zhang, Qi Wu, Congcong Liang, and Rui Fu for their technical support.

Disclosure of Interests: None declared

DOI: 10.1136/annrheumdis-2020-eular.5801

\section{\begin{tabular}{|l|l|l|l|}
\hline FRI0172 & THE INFLUENCE OF CALCINEURIN INHIBITORS
\end{tabular} ON DEVELOPMENT OF CANCER IN PATIENTS WITH SYSTEMIC LUPUS ERYTHEMATOSUS: A RETROSPECTIVE OBSERVATIONAL STUDY IN THE LUNA REGISTRY}

K. Ichinose ${ }^{1}$, T. Igawa ${ }^{1}$, M. Okamoto ${ }^{1}$, A. Takatani ${ }^{1}$, N. Yajima ${ }^{2}$, K. E. Sada ${ }^{3}$, R. Yoshimi ${ }^{4}$, Y. Shimojima ${ }^{5}$, S. Ono ${ }^{6}$, H. Kajiyama ${ }^{7}$, S. Sato ${ }^{8}$, M. Fujiwara ${ }^{9}$, A. Kawakami ${ }^{1}{ }^{1}$ Nagasaki University Graduate School of Biomedical Sciences, Department of Immunology and Rheumatology, Division of Advanced Preventive Medical Sciences, Nagasaki, Japan; ${ }^{2}$ Showa University School of Medicine, Division of Rheumatology, Department of Internal Medicine, Shinagawa-ku, Tokyo, Japan; ${ }^{3}$ Okayama University Graduate School of Medicine Dentistry and Pharmaceutical Sciences, Department of Nephrology, Rheumatology, Endocrinology and Metabolism, Okayama, Japan; ${ }^{4}$ Yokohama City University Graduate School of Medicine, Department of Stem Cell and Immune Regulation, Yokohama, Japan; ${ }^{5}$ Shinshu University School of Medicine, Department of Medicine (Neurology and Rheumatology), Matsumoto, Japan: ${ }^{6}$ Yokohama City University Medical Center, Center for Rheumatic Diseases, Yokohama, Japan; ${ }^{7}$ Saitama Medical University, Department of Rheumatology and Applied Immunology Faculty of Medicine, Saitama, Japan; ${ }^{8}$ Fukushima Medical University School of Medicine, Department of Rheumatology, Fukushima, Japan; ${ }^{9}$ Yokohama Rosai Hospital, Department of Rheumatology, Yokohama, Japan

Background: It has been reported that the incidence of cancer in patients with systemic lupus erythematosus (SLE) is higher than that in healthy individuals, but the findings are inconsistent ${ }^{1}$. In the transplantation field, a few studies indicated an association between the use of immunosuppressants and an increased risk of cancer ${ }^{2}$. Calcineurin inhibitors (CNIs), which include cyclosporine and tacrolimus, have been used for $>30$ years to treat renal and extrarenal manifestations of SLE, but the effects of exposure to CNIs among SLE patients have not been established.

Objectives: We investigated the incidence of various cancers (including cervical dysplasia) among SLE patients registered in the LUpus registry of NAtionwide institution (LUNA). We also investigate whether the registrants' exposure to CNIs increased the risk of cancer.

Methods: We calculated the standardized incidence ratio (SIR) of cancer among SLE patients based on the age-standardized incidence rate of cancer reported by Japan's Ministry of Health, Labour and Welfare. A multivariate analysis of the risk of cancer was performed using the covariates of age, smoking history, $\mathrm{CNI}$ treatment history, maximum steroid dose in the past, and Systemic Lupus International Collaboration Clinics/American College of Rheumatology Damage Index [SDI]) value (excluding the occurrence of cancer) at the time of the patient's registration.

Results: We studied 714 patients (663 females; $88.9 \%$ ). The median age at registry was 44 [interquartile range (IQR): 35-56] years. The median past max. steroid dose was $40 \mathrm{mg} /$ day (IQR: $30-60 \mathrm{mg} /$ day), and the SDI at registration was 1 (IQR 0-2). Smoking history was present in 248 patients (34.9\%), and 53 patients $(7.4 \%)$ experienced cancer complications. Gynecologic malignancies accounted for $71 \%$ of all cancers, including 12 cervical dysplasia cases. The standardized incidence rate of cancer in these SLE patients was $1.46(95 \% \mathrm{Cl}: 1.07-1.85, \mathrm{p}<0.01)$. The multivariate analysis showed that a $\mathrm{CNI}$ treatment history was not a risk factor for the development of cancer (OR $1.76,95 \% \mathrm{Cl}: 0.63-4.88, \mathrm{p}=0.30$ ). After the covariance was adjusted for the propensity score, the risk of cancer in the CNIs group was not increased compared to the non-CNIs group (adjusted OR 2.46 95\% Cl: 0.68-8.91, $\mathrm{p}=0.20$ ).

Conclusion: The incidence of cancer in SLE was higher in the LUNA cohor than in the general population. Our results suggest that $\mathrm{CNI}$ treatment for individuals with SLE is not a risk factor for the development of cancer. References:

[1] Ladouceur A. et.al, Expert Rev Clin Immunol. 2018 Oct;14(10):793-802. [2] Gutierrez-Dalmau A. et.al, Drugs 2007;67(8):1167-98.

Disclosure of Interests: None declared

DOI: 10.1136/annrheumdis-2020-eular.1379

FRI0173

IDENTIFICATION OF RISK FACTORS FOR DEVELOPMENT OF OSTEONECROSIS IN SYSTEMIC LUPUS ERYTHEMATOSUS

R. Kallas ${ }^{1}$, J. LI' ${ }^{1}$, M. Petri ${ }^{1} .{ }^{1}$ Johns Hopkins University School of Medicine, Baltimore, United States of America

Background: Patient with Systemic Lupus Erythematosus (SLE), particularly those who received corticosteroids are at a high risk of osteonecrosis (ON). 